\title{
Efectos de las terapias ecuestres en personas con parálisis cerebral
}

\section{Effects of equestrian therapies on people with cerebral palsy}

\section{Resumen}

El objetivo del artículo es revisar la literatura científica existente a fin de comprobar los efectos de las terapias ecuestres en personas con parálisis cerebral. Se realizó una revisión bibliográfica en las bases de datos de Medline (PubMed), Dialnet, Scopus, Sportdiscus y Web of Science, estableciendo como criterios de inclusión para seleccionar los estudios clínicos por revisar que estos hubieran sido publicados entre 2001 y 2016 , en castellano o inglés. Asimismo, se rechazaron aquellos en los que los sujetos recibieran una combinación de tratamientos farmacológicos. Se revisaron un total de 68 artículos, de los cuales 20 cumplieron los criterios de inclusión. Se analizaron la población objeto de estudio, el tipo de tratamiento, la temporalización y los resultados obtenidos. En general, se observan beneficios significativos en el uso de las terapias ecuestres como tratamiento para personas con parálisis cerebral.

\section{Palabras clave}

Terapias ecuestres, terapia asistida por caballos, hipoterapia, parálisis cerebral, tono muscular.

\begin{abstract}
This work aims to review the existing scientific literature in order to check the effects of equestrian therapies on people with cerebral palsy. A bibliographical review was performed in databases of Medline (PubMed), Dialnet, Scopus, Sportdiscus y Web of Science, establishing as inclusion criteria to select clinical studies for review that these had been published between 200I and 2016, in Spanish or English, likewise there were rejected those in which the subjects received a combination of pharmacological treatments. There were reviewed 68 articles in total, 20 of which met the inclusion criteria. There were analysed many criteria, such as the population object of the study, the type of treatment, the schedule and the results obtained. In general, significant benefits are observed in the use of equestrian therapies as a treatment for people with cerebral palsy.
\end{abstract}

\section{Keywords}

Equestrian therapies, equine assisted therapy, hippotherapy, cerebral palsy, muscle tone.

\section{Augusto Jiménez de la Fuente \\ <augusto.jdlf@gmail.com> \\ Universidad Politécnica de Madrid}

\section{Para citar:}

Jiménez, A. (20I7): "Efectos de las terapias ecuestres en personas con parálisis cerebral”. Revista Española de Discapacidad,

5 (2): I 7 I-I 84 .

Doi: <https://doi.org/IO. 5569/23405 I04.05.02.09>

Fecha de recepción: I4-I I-20I6

Fecha de aceptación: I9-IO-20I7 


\section{Introducción}

En la actualidad las terapias ecuestres son una metodología de rehabilitación cada vez más común dentro del tratamiento que reciben personas con distintos tipos de discapacidades, en este artículo vamos a centrarnos en los efectos de dichas terapias en las personas con parálisis cerebral, para lo cual se presenta una revisión bibliográfica de diferentes artículos científicos que avalan las terapias ecuestres como un tratamiento eficaz en la mejora de la calidad de vida de las personas con parálisis cerebral.

Para ello, antes de pasar a la revisión bibliográfica consideramos importante definir en profundidad los dos conceptos principales en torno a los cuales gira este artículo, la parálisis cerebral y las terapias ecuestres.

\section{¿Qué es la parálisis cerebral?}

La primera definición consensuada de la parálisis cerebral (PC) data de 1958 , como un trastorno motor persistente que aparece antes de los tres años, debido a una interferencia no progresiva en el desarrollo del cerebro que tiene lugar antes de que el crecimiento del SNC se complete (MacKeith y Polani, I958).

Dicha definición ha ido evolucionando en función de diversos criterios etiológicos, funcionales y clínicos hasta describir la PC como un grupo de trastornos del desarrollo del movimiento y de la postura, que causan limitación en la actividad y son atribuidos a alteraciones no progresivas que ocurren en el cerebro durante el desarrollo del feto o del niño pequeño, acompañándose con frecuencia con trastorno motor, alteraciones de la sensibilidad, cognición, comunicación, percepción, comportamiento y/o crisis epilépticas (Bax et al., 2005).

Por tanto, podemos definir la PC como un grupo de trastornos que afecta principalmente al desarrollo del movimiento y la postura, causando limitación de la actividad, y suele ir acompañada frecuentemente por trastornos sensoriales, perceptivos, cognitivos, de comunicación, del comportamiento, epilepsia y de problemas musculoesqueléticos secundarios debidos a una lesión neurológica no progresiva producida durante la gestación o en el nacimiento y persistente a través de la esperanza de vida (Rosenbaum et al., 2007).

La parálisis cerebral es la patología más frecuente de discapacidad física en la edad pediátrica (Kuban y Leviton, I994; López Ros et al., 2003) y el principal motivo de discapacidad física grave (Hutton y Pharoah, 2006), con una prevalencia de 2 a 2,5 casos por I.000 nacimientos (Cans, 2000), con ligero predominio del sexo masculino, y una mayor incidencia en América respecto a Europa.

Centrándose en España, Camacho Salas et al. (2007) subrayan la dificultad para establecer datos epidemiológicos acerca de la prevalencia de la PC. Según estos autores y basándose en la Encuesta sobre Discapacidades, Deficiencias y Estado de Salud 1999 (Instituto Nacional de Estadística, Instituto de Migraciones y Servicios Sociales, Fundación ONCE, 2002), se estima que había casi 60.000 personas mayores de seis años con diagnóstico de PC en España en el año I999; a su vez se observa en la Encuesta de Discapacidad, Autonomía personal y situaciones de Dependencia 2008 (Instituto Nacional de Estadística, 2009) cómo las causas congénitas o los problemas en el momento del parto (dentro de los cuales podríamos encuadrar a la PC) provocan fundamentalmente deficiencias mentales en unas I 26.000 personas en España.

Existen múltiples causas que originan el daño cerebral, entre las que se encuentran: el desarrollo defectuoso del cerebro, la anoxia, la prematurez, la hipoglucemia, causas genéticas, la hemorragia intracraneal, incompatibilidad de Rh, la excesiva ictericia neonatal, el traumatismo y la infección. En algunos casos, la causa no está clara y, en muchos otros, el hecho de conocerla no indica necesariamente un diagnóstico o historia del caso (Franco, 20I2). 
La clasificación de tipos de parálisis cerebral varía según clínica, pero generalmente, los tipos más comunes son el espástico (del $70 \%$ al 80 $\%$ de los casos), el atetoide y el atáxico (del $5 \%$ al ıo \% de los pacientes) (Martínez Espinosa y Morey Tomás, I998; Levitt, 2002; López Ros et al., 2003).

Desde I990 se ha producido un aumento de la esperanza de vida en las personas con parálisis cerebral, incluso en aquellas con problemas funcionales más graves (Hutton y Pharoah, 2002, 2006). Aunque algunas pueden vivir hasta alcanzar la década de los noventa, la esperanza de vida media en las personas con parálisis cerebral es de unos 34 años (Schutt, I993). La utilización de antibióticos ha permitido que niños con PC grave, especialmente vulnerables a las infecciones respiratorias, alcancen la edad adulta. Así como la posibilidad de alimentar a los niños mediante sonda nasogástrica o gastrostomía ha contribuido a evitar la malnutrición en niños con disfagia u otros problemas digestivos (Camacho Salas et al., 2007).

Respecto al ámbito intelectual, más del $50 \%$ de los niños con PC tienen un Cociente Intelectual (CI) normal, siendo más afectados aquellos niños con tetraparesia y menos afectados el grupo de niños con hemiparesia. Los niños con mejor CI se agrupan en aquellos con mejor funcionalidad motora o más bien aquellos niños con mejor funcionalidad motora tienen mejor CI. Por otro lado, los niños con PC tetraparética tienen un grado de compromiso intelectual mayor que los niños con hemiplejia (Paneth y Stark, I983; Murphy et al., I998; Beckung y Hagberg, 2002; Ashwal, S. et al., 2004). Así se resume que aquellos niños con menor afectación motora presentan a su vez un mayor CI.

\section{¿Qué son las terapias ecuestres?}

Las terapias ecuestres son cada vez una intervención terapéutica más conocida y las terapias asistidas con caballos son más demandadas tanto en el sector de la educación física, la recreación y el turismo, pero es en el ámbito de la salud donde más popularidad e importancia están conquistando (Cynarski y Obodynski, 2008).

En base a las definiciones de diferentes autores (American Hippotherapy Association, 20I0; Ernst y de la Fuente, 2007; Gross, 2006; Hernández y Luján, 2006; Arias et al., 2008; Villasana et al., 20I I) y tratando de ofrecer una perspectiva global de los diferentes aspectos contemplados y complementarios de las definiciones de dichos autores acerca de las terapias ecuestres, consideramos que estas consisten en un tipo de terapia física en la que se aprovechan los principios del caballo (movimientos del caballo, temperatura corporal...) para tratar diferentes tipos de afecciones (físicas, psicológicas, sensoriales...) que hacen que la persona no pueda ejercer ningún tipo de acción sobre el caballo y lograr una rehabilitación funcional y psicológica, así como una mejora de las aptitudes básicas y de la adaptación social.

Este conjunto de técnicas rehabilitadoras se puede aplicar a un gran número de discapacidades, aunque fundamentalmente son las del aparato locomotor, por lo que se podría decir que va dirigida principalmente a personas con discapacidades físicas, ya sean congénitas o adquiridas.

La elección como objeto de estudio de los efectos de las terapias ecuestres en personas con $\mathrm{PC}$, radica en el carácter innovador de estas y en los beneficios que aportan a las personas con discapacidad que las realizan, los cuales vienen avalados en los últimos años por el incremento de investigaciones científicas sobre dichas terapias complementarias a las tradicionales y el aumento del número de centros que ofertan dichas terapias asistidas con caballos, así como de instituciones educativas que imparten formación al respecto.

En la actualidad existen programas de terapias ecuestres en Inglaterra, Francia, Italia, Alemania, Bélgica, Dinamarca, Finlandia, Holanda, Noruega, Suecia, Austria, España y Portugal (Engel, I998), aunque en diverso grado de 
evolución. El progresivo auge de las terapias ecuestres y su paulatina profesionalización ha llevado a su reconocimiento por parte de organizaciones como la OMS, que, en sus últimas recomendaciones, incluye la equitación como actividad deportiva beneficiosa para personas con patologías físicas, psíquicas y sociales (Ernst y de la Fuente, 2007), o como la American Physical Therapy Association y la American Occupational Therapy Association, que indican los efectos terapéuticos de la equitación (Scott, 2005).

En España existen programas de terapias ecuestres en todas las comunidades autónomas, pero lamentablemente aún son pocos los que siguen las normas de funcionamiento, buena práctica y cualificación profesional aceptadas internacionalmente. Esta escasez de programas con metodología de trabajo adecuada puede deberse a varios factores: en primer lugar, a la relativa "juventud" de las terapias ecuestres en España. En segundo, y hasta donde se tiene constancia, a la no existencia de una federación nacional o colegio profesional fuerte que, a la manera de países como Estados Unidos, Inglaterra o Francia (Engel, I998), aglutine a la mayor parte de centros de terapia del país, marque estándares de calidad en la práctica y exija cualificación profesional suficiente a sus afiliados como condición previa al ejercicio de la terapia.

\section{Características generales de las terapias ecuestres}

La base de las terapias ecuestres se encuentran en el manejo del movimiento multidimensional del caballo combinado con diferentes técnicas rehabilitatorias (Haskin et al., I982), sensoperceptivas (Haskin et al., I974), auditivas (McCullough y Martin, 2005), motoras y visuales (Mackinnon et al., I995) entre otras, contribuyendo de forma positiva a la estimulación y desarrollo en áreas como: cognitiva, física, psíquica, emocional, social, psicomotriz de las personas sea que presenten alguna deficiencia en sus capacidades o afecciones en alguna de las áreas mencionadas (Mackay et al., I988). Es por esto que dichas terapias tienen una aplicación terapéutica en medicina, psicología, psiquiatría y pedagogía.

Con los elementos que ofrece la monta y el trato con el caballo y su entorno, el terapeuta entrenado puede trabajar sobre muchos objetivos distintos, desde la rehabilitación física, la psicomotricidad y la integración sensorial, hasta el entrenamiento en habilidades sociales y la modificación de conducta.

Las terapias ecuestres se basan en tres principios (American Hippotherapy Association, 20I0; Arias et al., 2008; Caudet, 2002; Engel, I998; Ernst y de la Fuente, 2007; Gross, 2006; Hernández y Luján, 2006; Scott, 2005):

- Transmisión de calor corporal del caballo al cuerpo de la persona.

- Transmisión de impulsos rítmicos del dorso del caballo al cuerpo de la persona.

- Transmisión de un patrón de locomoción equivalente al patrón fisiológico de la marcha humana.

\section{Objetivo}

Revisar la literatura científica existente a fin de comprobar los efectos de las terapias ecuestres en personas con parálisis cerebral, partiendo de la hipótesis de que un programa de terapias ecuestres disminuye la intensidad de la espasticidad y la extensibilidad de los músculos aductores de caderas, mejora el equilibrio en sedestación y el patrón de la marcha y se da un aumento de la autopercepción en personas con parálisis cerebral.

\section{Método}

Se realizó una revisión bibliográfica en las bases de datos de Mediline (PubMed), Dialnet, 
Scopus, Sportdiscus y Web of Science, de los principales estudios publicados en los que se investigara la eficacia de las terapias ecuestres como tratamiento para las personas con PC. Las frases de búsqueda estuvieron compuestas por los términos "hipoterapia OR hippotherapy" o "terapias ecuestres $O R$ equestrian therapies" o "terapias asistidas por caballos $O R$ equine assisted therapy" y "parálisis cerebral $O R$ cerebral palsy".

Esta revisión se realizó siguiendo el procedimiento de búsqueda sistemático propuesto por Peinado et al. (2007), en el que se crea una frase de búsqueda con palabras clave que se introduce en las principales bases de datos, nacionales e internacionales, del área de estudio.

Los criterios de inclusión para seleccionar los estudios clínicos por revisar fueron los siguientes: publicados entre 200 I y 2016 , en castellano o inglés; así mismo, se rechazaron aquellos en los que los sujetos recibieran una combinación de tratamientos farmacológicos.

Tras obtener los resultados de las diferentes búsquedas, se aplicó un análisis cualitativo de los artículos mediante la lectura en su versión completa y se procedió a la selección de artículos con los criterios de inclusión preestablecidos. También se revisaron las referencias de los artículos seleccionados con el fin de obtener nuevos trabajos potencialmente interesantes.

De todos los artículos encontrados tras la búsqueda bibliográfica, un total de 68, tan solo se hará mención a 20 estudios clínicos, que cumplieron los citados criterios de inclusión. Los resultados de todos los estudios clínicos seleccionados mostraron mejoras significativas respecto a los valores iniciales o respecto al grupo control. A continuación, se presentan en orden cronológico los diferentes artículos seleccionados con las características generales de los programas de hipoterapia, los sujetos a los que iban dirigidos y los resultados obtenidos.

\section{Resultados}

Sterba et al. (2002) determinaron en un estudio ciego aplicando la Gross Motor Function Measure (GMFM), los efectos de las terapias ecuestres en I7 niños con parálisis cerebral (diplejía espástica, tetraplejia espástica y hemiplejia espástica) con una media de edad de 9 años y ro meses. Las sesiones de terapia ecuestre tuvieron una temporalidad de I hora por semana durante i 8 semanas. Al final de dicho periodo se comprobó una mejora en la GMFM, lo que según los autores sugiere que las terapias ecuestres pueden mejorar la función motora gruesa en niños con parálisis cerebral, lo cual puede reducir el grado de discapacidad motora.

Benda et al. (2003) evaluaron los efectos de un programa de hipoterapia de 8 minutos en un grupo de I 5 niños entre 4 y I 2 años con parálisis cerebral espástica analizando la modificación de la actividad electromiográfica de la musculatura del tronco y de los muslos al sentarse, pararse y caminar, antes y después de la intervención. Tras esta se mostraron cambios significativos en la musculatura, aumentando la simetría de los grupos musculares.

Casady y Nichols-Larsen (2004) comprobaron si un programa de Io sesiones de hipoterapia con una alternancia semanal tendría efectos en el desarrollo general funcional de ro niños con parálisis cerebral con edades entre los 2 y los 6 años. Para ello aplicaron dos pre-test y dos posttest usando la Pediatric Evaluation of Disability Inventory (PEDI) y la GMFM, comparando los resultados antes y después del programa de hipoterapia. Los resultados demostraron mejoras significativas entre las medidas pre-test y post-test lo que sugiere que la hipoterapia tiene efectos positivos en el desarrollo de las habilidades motoras de niños con PC.

Debuse et al. (2005) diseñaron y pasaron un cuestionario en el que evaluaban los efectos principales percibidos por los fisioterapeutas de personas con parálisis cerebral que realizaban 
sesiones de hipoterapia en Alemania y el Reino Unido y cómo dichas sesiones de hipoterapia eran llevadas a cabo. Dichos autores observaron diferencias considerables en cómo la hipoterapia era practicada en Reino Unido y Alemania. A pesar de esto, el estudio reveló semejanzas en los efectos percibidos entre los pacientes con parálisis cerebral, concretamente, una mejora del control de tono de músculo, mejora del control postural y beneficios psicológicos. Los resultados también indican un escaso uso de medidas de resultado para evaluar dichos efectos.

Fuertes y Hernández (2005) evaluaron el efecto de un programa de hipoterapia en la actividad muscular en niños con parálisis cerebral espástica. Para ello seleccionaron I 5 niños de entre 4 y I 2 años de edad y se les midió mediante electromiograma la actividad muscular del tronco y grupos musculares proximales de las extremidades inferiores durante la sedestación, bipedestación y durante la marcha, antes y después de recibir un tratamiento de 8 minutos de hipoterapia, observando una mejoría significativa en la simetría de la actividad de los grupos musculares que presentaban mayor asimetría antes de la sesión.

Debuse et al. (2009) repitieron su estudio cualitativo de 2005 pero esta vez explorando la experiencia de la hipoterapia en personas con parálisis cerebral desde la perspectiva del usuario. Diecisiete usuarios de entre 4 y 63 años, con o sin sus padres, participaron en los grupos focales y entrevistas individuales en seis centros en Gran Bretaña y Alemania. El estudio concluyó que los principales efectos de la hipoterapia, identificados por los usuarios y los padres de estos, son la normalización del tono muscular, la mejora del control del tronco, la mejora de la capacidad de caminar, la mejora de la ejecución de las actividades de la vida diaria, y el aumento de la autoeficacia, la confianza y la autoestima.

McGibbon et al. (2009) investigaron los efectos inmediatos de ro minutos de hipoterapia, en comparación con ro minutos de barril sentado, en la simetría de la actividad del músculo aductor durante la marcha en 47 niños con
PC en una primera fase, para posteriormente investigar a largo plazo los efectos de I 2 semanas de una sesión semanal de hipoterapia sobre la actividad de los aductores, de la función motora gruesa utilizando la GMFM, y el autoconcepto en una segunda fase en 6 niños con PC. Los resultados mostraron una mejora significativa en la simetría de aductores en la fase primera, así como en la segunda fase una mejora significativa tanto en la simetría de aductores, como en la GMFM y en los sentimientos de auto-competencia.

Shurtleff et al. (2009) estudiaron si un programa de intervención de I 2 semanas de hipoterapia mejora la estabilidad del tronco y la cabeza y la extensión y orientación de las extremidades superiores en I I niños con PC espástica dipléjica con edades comprendidas entre los 5 y I 3 años. Los resultados mostraron mejoras significativas en la estabilidad de la cabeza y el tronco, así como en la extensión y orientación de las extremidades superiores tras las I 2 semanas de intervención de hipoterapia. Inclusive manteniéndose dichos cambios después de un período de I 2 semanas.

Castillo y Quintero (2010) comprobaron la influencia de un tratamiento de hipoterapia en la función motora gruesa de un niño con parálisis cerebral espástica. Para ello diseñaron un programa de hipoterapia de 40 sesiones, cada una con una duración de dos horas y realizadas cinco días a la semana durante un mes. Para comprobar los cambios, aplicaron previo y post al programa de hipoterapia la GMFM, observando un incremento en la integración del hemicuerpo izquierdo acompañado de una adecuada disociación escapular y pélvica y una disminución de la rigidez del cinturón pélvico; al mismo tiempo, se verificó una mejoría del control cefálico, un fortalecimiento de la musculatura flexora cervical, así como de los músculos paraespinales.

Honkavaara y Rintala (2010) investigaron los efectos a corto plazo de un programa de hipoterapia en la marcha de niños con parálisis cerebral. Los participantes fueron dos niños de I 2 y I 3 años con diplejía espástica y una niña 
de I4 años con parálisis cerebral atetoide. Se determinaron los cambios cuantitativos en los parámetros funcionales de la marcha (velocidad, longitud de zancada y la cadencia) después de tres semanas de hipoterapia (nueve sesiones). Los dos muchachos demostraron una mejoría en la longitud de zancada y la velocidad de la marcha sin una mejora sostenida en la cadencia. En la chica no hubo un aumento en la longitud de zancada ni en la cadencia, pero sí en la velocidad. Por tanto, los resultados indicaron que es posible que la hipoterapia a corto plazo pueda mejorar la marcha funcional en niños con parálisis cerebral.

Kwon et al. (20II) evaluaron los efectos de la hipoterapia centrándose en parámetros cinemáticos de la pelvis y la cadera durante la marcha en 32 niños con parálisis cerebral espástica bilateral. Para ello sometieron a los niños a un programa de hipoterapia con sesiones de 30 minutos dos veces a la semana durante 8 semanas consecutivas. Los resultados que obtuvieron es que dicho programa de hipoterapia mejoró significativamente la velocidad al caminar, la longitud de zancada y la cinemática de la pelvis (promedio de la inclinación pélvica anterior, la inclinación pélvica anterior en el contacto inicial, la inclinación pélvica anterior en posición terminal). Por tanto, concluyeron que la hipoterapia, gracias al movimiento multidimensional del caballo, puede ser utilizada en conjunción con la terapia física estándar para la mejora de la marcha y el equilibrio en los niños parálisis cerebral espástica bilateral.

Chang et al. (2012) investigaron si la hipoterapia podía mejorar el rendimiento funcional de niños en edad preescolar y escolar con parálisis cerebral espástica bilateral. Para ello se seleccionaron 33 niños mayores de cuatro años con parálisis cerebral espástica bilateral. Los niños recibieron 30 minutos de hipoterapia dos veces por semana durante ocho semanas consecutivas. Se evaluaron la motricidad gruesa y los valores de la escala del balance pediátricos durante el período de control de pre-montar a caballo, en el inicio de la hipoterapia y después del programa de hipoterapia. Los resultados mostraron mejoras significativas tras el programa de hipoterapia, mejorando la función motora gruesa y el equilibrio en los pacientes con parálisis cerebral pediátricos sin efectos adversos.

El-Meniawy y Thabet (20I2) examinaron los efectos de un programa de hipoterapia en treinta niños con parálisis cerebral espástica, de edades comprendidas entre seis y ocho años. El programa consistía en doce sesiones de hipoterapia repartidas en una sesión semanal. Dividieron aleatoriamente a los niños en dos grupos de igual número, un grupo de estudio y otro grupo de control, y se evaluaron los parámetros geométricos de cada niño de los dos grupos antes y después de los tres meses de tratamiento. El grupo control recibió un programa de ejercicios, mientras que el grupo estudio recibió el tratamiento de hipoterapia, además del mismo programa de ejercicios que el grupo A. Al finalizar el programa de hipoterapia observaron una mejoría significativa en todas las variables de medición de los dos grupos, pero con una mayor mejoría en el grupo que había realizado las sesiones de hipoterapia.

Herrero et al. (2012) investigaron si la hipoterapia (cuando se aplica por un simulador) mejora el control postural y el equilibrio en los niños con parálisis cerebral. Para ello seleccionaron treinta y ocho niños de entre 4 y I 8 años de edad con parálisis cerebral. Los participantes fueron asignados aleatoriamente en un grupo intervención (simulador $\mathrm{ON}$ ) o control (simulador OFF). El tratamiento se realizó una vez a la semana, con una duración de I 5 minutos, durante ro semanas. Llevaron a cabo una evaluación al inicio del estudio (antes de la aleatorización), al final de la intervención y I 2 semanas después de completar la intervención. Los resultados mostraron una mejora en el equilibrio sentado tras el programa de hipoterapia, pero dicha mejoría no se mantuvo tras las I 2 semanas desde la intervención lo que nos indica que si queremos que la mejoría se mantenga es necesario mantener el programa de hipoterapia de forma continuada.

Kang et al. (2OI2) comprobaron los efectos de la hipoterapia sobre el equilibrio sentado en 45 
niños con parálisis cerebral. Para ello dividieron de forma randomizada a los niños en tres grupos, un grupo control $\left(\mathrm{n}=\mathrm{I}_{5}\right)$ que no recibiría ningún tipo de tratamiento, un grupo que solo realizaría hipoterapia $\left(\mathrm{n}=\mathrm{I}_{5}\right.$ ) y otro grupo que solo realizaría ejercicios de fortalecimiento y estiramiento por parte de fisioterapeutas $(\mathrm{n}=\mathrm{I} 5)$, ambos grupos en sesiones semanales de 30 minutos durante ocho semanas. Tras las ocho semanas de intervención, comprobaron que los movimientos de balanceo disminuyeron en el grupo que recibió hipoterapia respecto al que recibió fisioterapia, así como este grupo también mejoró significativamente respecto al grupo control. Por lo tanto, concluyen que un tratamiento de hipoterapia mejora el equilibrio sentado en niños con parálisis cerebral y combinado con un tratamiento de fisioterapia se logran aún mejores resultados.

Baik et al. (20I4) comprobaron los efectos de montar a caballo en el tono muscular de las extremidades pélvicas y los rangos de movimiento articular en 16 niños con parálisis cerebral espástica. Midieron el tono muscular mediante la Modified Ashworth Scale (MAS) y utilizaron un goniómetro para medir el rango de movimiento (ROM). El programa de equitación terapéutica que llevaron a cabo consistió en 2 sesiones semanales de 60 minutos al día, durante un total de $\mathbf{2} 2$ semanas. Los resultados mostraron una diferencia estadísticamente significativa en el tono muscular de la rodilla de los niños con PC (P <O,OI). La participación en el programa de equitación terapéutica también mostró una diferencia estadísticamente significativa en el rango de movimiento articular de la cadera y de la rodilla de los niños con PC (P<O,OI). Los autores concluyen que los efectos de la monta terapéutica en niños con parálisis cerebral espástica son positivos y, por tanto, las terapias ecuestres han de formar parte de la rehabilitación requerida para niños con parálisis cerebral espástica.

Park et al. (20I4) investigaron los efectos de la hipoterapia en la función motora gruesa y el rendimiento funcional en 34 niños con parálisis cerebral espástica, con edades entre $\operatorname{los} 3$ y I 2 años. Estos se sometieron a sesiones de hipoterapia de 45 minutos de duración, dos veces a la semana durante 8 semanas. Veintiún niños con parálisis cerebral espástica fueron reclutados para el grupo de control con una distribución no significativa de la función motora gruesa y la edad media respecto al grupo de intervención. Como instrumentos de evaluación se utilizaron la GMFM-66, GMFM88 y la PEDI, las cuales se aplicaron antes del tratamiento y después de 8 semanas de la intervención. Las puntuaciones en las GMFM y en la PEDI-SFS mejoraron significativamente en ambos grupos tras las ocho semanas de hipoterapia, de forma significativa en el grupo de intervención respecto al grupo control. De este modo, los autores demuestran los efectos beneficiosos de la hipoterapia en la función motora gruesa y el rendimiento funcional en niños con PC.

Antunes et al. (2016) exploraron los efectos inmediatos de un protocolo de hipoterapia usando un ritmo de paso-trote en los parámetros espaciotemporales de la marcha y el tono muscular en Io niños con parálisis cerebral espástica bilateral. Los niños con PC se sometieron a dos sesiones de hipoterapia por semana. Se aplicaron dos protocolos de 30 minutos de duración en días separados: en el Protocolo I el ritmo del caballo era de paso y en el Protocolo 2 el ritmo del caballo era un ritmo de paso-trote. Finalmente observaron una mejoría en todos los parámetros espaciotemporales, excepto para la velocidad de la marcha. La espasticidad de los músculos aductores de la cadera se redujo significativamente como resultado inmediato de ambos protocolos, pero esta disminución fue más evidente después del ritmo de pasotrote. Los autores firman que el protocolo de paso-trote es factible y es capaz de inducir un efecto inmediato que mejora los parámetros espaciotemporales de la marcha y de la espasticidad en los músculos aductores de la cadera en niños con PC.

Champagne et al. (2016) evaluaron los efectos de la hipoterapia en las capacidades físicas de trece niños con PC de entre 4-I 2 años clasificados en un Nivel I o II de la GMFM. 
Los participantes recibieron Io semanas de hipoterapia, con una sesión semanal de 30 minutos de duración. La función motora gruesa y la habilidad se midieron con la forma corta de Bruininks-Oseretski Motor Proficiency (Bot2-SF) y mediante la GMFM-88, dos veces antes de empezar el programa (TI y TI'), inmediatamente después ( $\mathrm{T} 2$ ), y Io semanas después del final del programa $\left(\mathrm{T}_{3}\right)$. Los resultados mostraron una mejoría entre las puntuaciones medias de inicio e inmediatamente después de la intervención; las puntuaciones medias inmediatamente después de las sesiones de hipoterapia y ıo semanas después de la intervención no fueron diferentes. Los autores concluyen que un programa de hipoterapia durante ro semanas puede mejorar las funciones corporales y la ejecución de actividades de motricidad gruesa y fina en los niños con PC.

Moraes et al. (2016) evaluaron los efectos de I 2 y 24 sesiones de hipoterapia en el equilibrio postural sentado, el equilibrio dinámico, y el rendimiento funcional en I 5 niños con PC con edades comprendidas entre los 5 y io años. El protocolo de hipoterapia se realizó durante 30 minutos, dos veces a la semana, durante I 2 semanas, midiendo el equilibrio postural en posición sentada utilizando una plataforma de fuerza AMTI AccuSway Plus I semana antes de iniciar el programa de hipoterapia y después de 12 y 24 semanas. La Berg Balance Scale (BBS) y PEDI se utilizaron antes y después de las 24 sesiones. Los autores observaron diferencias significativas para las variables centro de presión $(\mathrm{CP})$, incluyendo medio-lateral (COPml), el desplazamiento anteroposterior (COPAP), y la velocidad de desplazamiento (VelCOP), especialmente después de 24 sesiones. También hubo diferencias significativas en las puntuaciones de la BBS y un aumento en la puntuación PEDI asociados con habilidades funcionales (autocuidado, función social y movilidad). Por lo tanto, concluyen que un programa de hipoterapia mejora el equilibrio postural en la posición sentada, el equilibrio dinámico, y la funcionalidad en niños con PC, especialmente después de 24 sesiones de hipoterapia.

\section{Discusión}

Todos los estudios clínicos muestran que la aplicación de un programa de terapias ecuestres en personas o niños con parálisis cerebral provoca mejoras en la reducción de la espasticidad muscular en miembros inferiores, una mejora del control postural de cabeza y tronco, una mejora del equilibrio tanto sentado como en bipedestación y durante la marcha, una mejora en la ejecución de habilidades motrices gruesas y finas, una mejora en la simetría de grupos musculares así como un aumento del sentimiento de auto-eficacia, confianza y autoestima.

Los resultados obtenidos en esta revisión permiten afirmar que la literatura científica sobre los efectos del uso de las terapias ecuestres o de la hipoterapia en el tratamiento de personas con parálisis cerebral es abundante y objeto de investigación actual. Además, dada la diversidad y número de sujetos analizados tanto por el tipo de parálisis cerebral (espástica, atetoide) como por los rangos de edad que abarcan, desde los 2 hasta los I 8 años, nos permiten generalizar los resultados a personas con la misma patología y el mismo nivel de gravedad, en especial a niños con parálisis cerebral espástica, que componen el grueso de la población objeto de estudio.

También podemos comprobar como la Gross Motor Function Measure (GMFM) y la Pediatric Evaluation of Disability Inventory (PEDI) son los instrumentos más empleados para la valoración de la función motora en niños con parálisis cerebral, en este caso a la hora de comprobar los efectos de las terapias ecuestres, pero también de forma general aplicando otro tipo de tratamientos o terapias (Ketelaar et al., I998; Vos-Vromans et al., 2005).

Respecto a la temporalización de las sesiones de hipoterapia o terapia ecuestre no podemos establecer una temporalidad en concreto. Los estudios abarcan desde sesiones de 8 minutos de duración hasta las 2 horas; con una periodicidad diaria, dos veces por semana o una sesión 


\begin{tabular}{|c|c|c|c|c|c|c|}
\hline Edad & Tipo PC & $\begin{array}{l}\text { Instrumentos } \\
\text { utilizados }\end{array}$ & $\begin{array}{l}\text { Duración } \\
\text { sesiones }\end{array}$ & $\begin{array}{l}\text { Periodicidad } \\
\text { sesiones }\end{array}$ & $\begin{array}{l}\text { Duración } \\
\text { programa }\end{array}$ & Mejoras observadas \\
\hline $2-18$ & Espástica & $\begin{array}{l}\text { Gross Motor } \\
\text { Function Measure } \\
\text { (GMFM) } \\
\\
\\
\text { Pediatric } \\
\text { Evaluation } \\
\text { of Disability } \\
\text { Inventory } \\
\text { (PEDI) }\end{array}$ & $\begin{array}{l}\text { Desde } 8 \\
\text { minutos } \\
\text { hasta } 2 \\
\text { horas }\end{array}$ & $\begin{array}{l}\text { Diaria } \\
\text { Semanal } \\
\text { Dos veces } \\
\text { por semana }\end{array}$ & $\begin{array}{l}\text { De } 4 \text { a } 18 \\
\text { semanas }\end{array}$ & $\begin{array}{l}\text { - Reducción de la } \\
\text { espasticidad muscular } \\
\text { en miembros inferiores. } \\
\text { - Mejora del control } \\
\text { postural de cabeza y } \\
\text { tronco. } \\
\text { - Mejora del equilibrio } \\
\text { tanto sentado como en } \\
\text { bipedestación y durante } \\
\text { la marcha. } \\
\text { - Mejora en la ejecución } \\
\text { de habilidades motrices } \\
\text { gruesas y finas. } \\
\text { Mejora en la simetría de } \\
\text { grupos musculares. } \\
\text { - Aumento del } \\
\text { sentimiento de } \\
\text { autoeficacia, confianza } \\
\text { y autoestima }\end{array}$ \\
\hline
\end{tabular}

Fuente: elaboración propia.

semanal; y siendo el estudio de menor duración de 4 semanas y el de mayor duración de 18 , estableciendo entre las 8 y las $\mathrm{I} 2$ semanas el tiempo de aplicación medio más estudiado.

\section{Conclusiones}

En este artículo se ha procurado definir las características de la parálisis cerebral y de las terapias ecuestres en profundidad, basándonos en artículos científicos, y en especial, haciendo referencia a aquellos artículos originales que definieron por primera vez las características de ambas. Esto lo consideramos relevante, en especial respecto a las terapias ecuestres, puesto que son muchas las definiciones y la forma de entender dicho tipo de terapias desde un ámbito no científico, y consideramos que solo dándoles la relevancia científica que se merecen, las terapias ecuestres ganarán en importancia y credibilidad tanto por parte de las familias afectadas con miembros con parálisis cerebral, por parte del mundo médico y rehabilitador, así como de las asociaciones, fundaciones, hípicas y demás que realizan o afirman realizar dicho tipo de terapias, la mayoría de ellas sin una base científica y con personal sin cualificar.

La aplicación de terapias ecuestres en personas con parálisis cerebral son un ejemplo de actividad físico-deportiva eficaz y beneficiosa, actuando en la persona tanto en su esfera social, física e intelectual; siempre y cuando estas se apliquen por personal cualificado y contando con las instalaciones, material y caballos adecuados para poder desarrollarlas.

En el apartado de referencias bibliográficas se puede observar como la gran mayoría de artículos citados han sido publicados en revistas de impacto internacional y los estudios han sido realizados por instituciones extranjeras, lo cual demuestra la falta de investigaciones nacionales en el ámbito de las ciencias de la actividad física y el deporte, al menos desde el ámbito de la salud y la discapacidad, y más concretamente en el de las terapias ecuestres. 
Una de las carencias observadas en los estudios sobre terapias ecuestres en personas con parálisis cerebral es la escasez de información sobre los ejercicios que en concreto se realizaban durante las sesiones de hipoterapia, aunque especifican la temporalización de las sesiones y la duración de estas, no se especifica en detalle la estructura de las sesiones y los objetivos y contenidos trabajados en ellas. En relación al principal instrumento empleado para llevar a cabo este tipo de terapias, el caballo, se hace referencia meramente a los tipos de aire (paso o trote), pero no se hace referencia a las características del caballo de terapias a nivel de razas o morfología. También observamos una escasez de información en cuanto al perfil del terapeuta ecuestre, ya que en muchas ocasiones observamos como este tipo de terapias son llevadas a cabo por personas sin ningún tipo de conocimientos en el ámbito de la discapacidad ni mucho menos del deporte y la actividad física desde la perspectiva salud. 
Referencias bibliográficas

American Hippotherapy Association (2010): Introducción a la hipoterapia (en línea). < http://www.americanhippotherapyassociation. org>, acceso I 4 de octubre de 2016.

Antunes, F. N. et al. (2016): "Different horse's paces during hippotherapy on spatio-temporal parameters of gait in children with bilateral spastic cerebral palsy: A feasibility study". Research in Developmental Disabilities, 59: 65-72.

Arias, V. et al. (2008): “Terapia asistida por caballos: nueva propuesta de clasificación, programas para personas con discapacidad intelectual y buenas prácticas”. Revista Española sobre Discapacidad Intelectual, 39 (2): 226, I 8-30.

Ashwal, S. et al. (2004): "Practice Parameter: Diagnostic assessment of the child with cerebral palsy Report of the Quality Standards Subcommittee of the American Academy of Neurology and the Practice Committee of the Child Neurology Society". Neurology, 62 (6): $85 \mathrm{I}-863$.

Baik, K. et al. (2OI4): “The effects of horseback riding participation on the muscle tone and range of motion for children with spastic cerebral palsy". Journal of exercise rehabilitation, I0 (5): 265 .

Bax, M. et al. (2005): "Executive Committee for the Definition of Cerebral Palsy. Proposed definition and classification of cerebral palsy, April 2005”. Developmental Medicine \& Child Neurology, 47: 57I-576.

Beckung, E. y Hagberg, G. (2002):

"Neuroimpairments, activity limitations, and participation restrictions in children with cerebral palsy". Developmental Medicine \& Child Neurology, 44 (5): 309-3 I6.

Benda, W. et al. (2003): "Improvement in muscle symmetry in children with cerebral palsy after equine-assisted therapy (hippotherapy)". Journal of Alternative and Complementary Medicine, 9 (6): 8I7-825.
Camacho Salas, A. et al. (2007): "Parálisis cerebral: concepto y registros de base poblacional”. Revista de Neurología, 45 (8): 503-508.

Cans, C. (2000): "Surveillance of cerebral palsy in Europe: a collaboration of cerebral palsy surveys and registers". Developmental Medicine \& Child Neurology, 42 (I2): 8I 6-824.

Casady, R.L. y Nichols-Larsen, D.S. (2004): “The effect of hippotherapy on ten children with cerebral palsy". Pediatric Physical Therapy, I 6 (3): I65-I72.

Castillo, K. y Quintero, J. G. (2010): Influencia de la hipoterapia en la función motora gruesa de un niño con parálisis cerebral espástica en una institución de la ciudad de Santiago de Cali: Estudio de caso (Trabajo Fin de Carrera). Colombia: Universidad Santiago de Cali.

Caudet, F. (2002): Equinoterapia: el caballo, mucho más que un amigo, Barcelona: Editorial Astri.

Champagne, D. et al. (2016): "Effect of hippotherapy on motor proficiency and function in children with cerebral palsy who walk". Physical \& occupational therapy in pediatrics, $\mathrm{I}-\mathrm{I} 3$.

Chang, H. J. et al. (2012): "The effects of hippotherapy on the motor function of children with spastic bilateral cerebral palsy". Journal of Physical Therapy Science, 24: I277-I 280.

Cynarski, W. J. y Obodynski, K. (2008): "HorseRiding in the physical education, recreation and tourism: Axiological reflection". Medsportpress, I4 (I): 37-43.

Debuse, D. et al. (2005): “An exploration of German and British physiotherapists' views on the effects of hippotherapy and their measurement". Physiotherapy theory and practice, 2 I (4): 2I9-242.

Debuse, D. et al. (2009): "Effects of hippotherapy on people with cerebral palsy from the users' perspective: A qualitative study". Physiotherapy Theory and Practice, 25 (3): I74-I92. 
El-Meniawy, G. y Thabet N. (201 2): "Modulation of back geometry in children with spastic diplegic cerebral palsy via hippotherapy training". Egyptian Journal of Medical Human Genetics, I3 (I): 63-7I.

Engel, B. T. (I998): Therapeutic riding I: Strategies for Instruction, Durango, CO: Barbara Engel Therapy Services.

Ernst, M. y de la Fuente, M. (2007): Manual básico de hipoterapia, Barcelona: La Liebre de Marzo.

Franco, A. B. (20I2): “Ayudas para la marcha en la parálisis cerebral infantil”. Revista Internacional de Ciencias Podológicas, 6 (I): 9-24.

Fuertes, M. y Hernández, J. (2005): Mejora de la simetría muscular en niños con parálisis cerebral mediante hipoterapia (Trabajo Fin de Postgrado), Barcelona: Sociedad Española de Fisioterapia en Pediatría.

Gross, E. (2006): Equinoterapia: La rehabilitación por medio del caballo, México: Trillas.

Haskin, M. et al. (I982): “The Pennsylvania horseback riding program for cerebral palsy”. American Journal of Physical Medicine \& Rehabilitation, 6I (3): I4I-I44.

Haskin, M. et al. (I974). "Therapeutic horseback riding for the handicapped". Archives of Physical Medicine and Rehabilitation, 55 (I0): 473-474.

Hernández, C. R. y Luján, J. (2006): "Equinoterapia; Rehabilitación holística". Plasticidad y Restauración Neurológica, 5 (I): 70-74.

Herrero, P. et al. (20I2): "Study of the therapeutic effects of a hippotherapy simulator in children with cerebral palsy: A stratified singleblind randomized controlled trial”. Clinical Rehabilitation, 26 (I2): I I05-III3.

Honkavaara, M. y Rintala, P. (2010): “The influence of short term, intensive hippotherapy on gait in children with cerebral palsy". European Journal of Adapted Physical Activity, 3 (2): 29-36.

Hutton, J. L., y Pharoah, P. O. (2002): "Effects of cognitive, motor, and sensory disabilities on survival in cerebral palsy". Archives of disease in Childhood, 86 (2): 84-89.

Hutton, J. L., y Pharoah, P. O. (2006): "Life expectancy in severe cerebral palsy". Archives of disease in Childhood, 9I (3): 254-258.

Instituto Nacional de Estadística (2009). Encuesta de Discapacidad, Autonomía Personal y Situaciones de Dependencia 2008 (EDAD), Madrid: INE.

Instituto Nacional de Estadística, Instituto de Migraciones y Servicios Sociales, Fundación ONCE (2002): Encuesta sobre Discapacidades, Deficiencias y Estado de Salud I999. Resultados detallados, Madrid: INE.

Kang, H. et al. (2OI 2): "Effects of hippotherapy on the sitting balance of children with cerebral palsy: A randomized control trial". Journal of Physical Therapy Science, 24 (9): 833-836.

Ketelaar, M. et al. (I998): "Functional motor abilities of children with cerebral palsy: a systematic literature review of assessment measures”. Clinical rehabilitation, I 2 (5):369380.

Kuban, K. C. K., y Leviton, A. (I994): “Cerebral palsy”. New England Journal of Medicine, 330 : I 88-I95.

Kwon, J. Y. et al. (20I I): "Effects of hippotherapy on gait parameters in children with bilateral spastic cerebral palsy". Archives of Physical Medicine and Rehabilitation, 92: 774-779.

Levitt, S. (2002): Tratamiento de la parálisis cerebral y del retraso motor, $3^{a}$ ed., $I^{a}$ reimp., Madrid: Médica Panamericana, D.L.

López Ros, P. et al. (2003): "Parálisis cerebral infantil”. Podología clínica, 4 (2): 56-64.

Mackay, I. et al. (I988): "Effects of therapeutic horseback riding on patients with multiple sclerosis: A preliminary trial". Physiotherapy Canada, 40 (2): 104-109.

MacKeith, R. C., y Polani, P. E. (I958): “Cerebral palsy". Lancet, I: 6I.

Mackinnon, J. et al. (I995): “A study of therapeutic effects of horseback riding for children with cerebral palsy". Physical and 
Occupational Therapy in Pediatrics, I 5 (I): I7-3I.

Martínez Espinosa, O. y Morey Tomás, C. (I998): "Alternativas ortopodológicas en las marchas neurológicas”. Revista española de podología, $9(7): 332-38 \mathrm{I}$.

McCullough, L. M. y Martin, I. (2005): Equine facilitated mental health: a field guide for practice, Texas: Legends Equestrian Therapy.

McGibbon, N.H. et al. (2009). Immediate and long-term effects of hippotherapy on symmetry of adductor muscle activity and functional ability in children with spastic cerebral palsy. Archives of Physical Medicine and Rehabilitation, 90 (6): 966-974.

Moraes, A. G. et al. (2016): "The effects of hippotherapy on postural balance and functional ability in children with cerebral palsy". Journal of Physical Therapy Science, 28 (8): 2220-2226.

Murphy, C. et al. (1998): "Epidemiology of mental retardation in children”. Mental Retardation and Developmental Disabilities Research Reviews, 4 (I): 6-I3.

Paneth, N. y Stark, R. I. (I983): “Cerebral palsy and mental retardation in relation to indicators of perinatal asphyxia". American Journal of Obstetrics \& Gynecology, I47 (8): 960-966.

Park, E. S. et al. (20I4): "Effects of hippotherapy on gross motor function and functional performance of children with cerebral palsy". Yonsei medical journal, 55 (6): I736-I742.
Peinado, P. J. B. et al. (2007): "La revisión bibliográfica sistemática en fisiología del ejercicio: recomendaciones prácticas". Revista Internacional de Ciencias del Deporte, 3 (6): 2-II.

Rosenbaum, P. et al. (2007): “A report: the definition and classification of cerebral palsy April 2006". Developmental Medicine \& Child Neurology, I09: 8-I4.

Schutt, A. (I993): "Cerebral Palsy”, en Sinaki, M.: Basic clinical rehabilitation medicine. San Luis: Mosby.

Scott, N. (2005): Special needs, special horses: A guide to the benefits of therapeutic riding, Denton: University of North Texas Press.

Shurtleff, T. L. et al. (2009): "Changes in dynamic trunk/head stability and functional reach after hippotherapy". Archives of physical medicine and rehabilitation, 90 (7): I I 85-II 95.

Sterba, J. A. et al. (2002): "Horseback riding in children with cerebral palsy: effect on gross motor function". Developmental Medicine \& Child Neurology, 44 (05): 301-308.

Villasana, G. et al. (20I I): "Evaluación de la hipoterapia en niños con trastornos del desarrollo psicomotor". Avances en Ciencia de la Salud, I (I): 25-29.

Vos-Vromans, D. C. et al. (2005): "Responsiveness of evaluative measures for children with cerebral palsy: the Gross Motor Function Measure and the Pediatric Evaluation of Disability Inventory". Disability and rehabilitation, 27 (20): I 245-I 252. 